\title{
LA-UR- $99-946$
}

Approved for public release; distribution is unlimited.
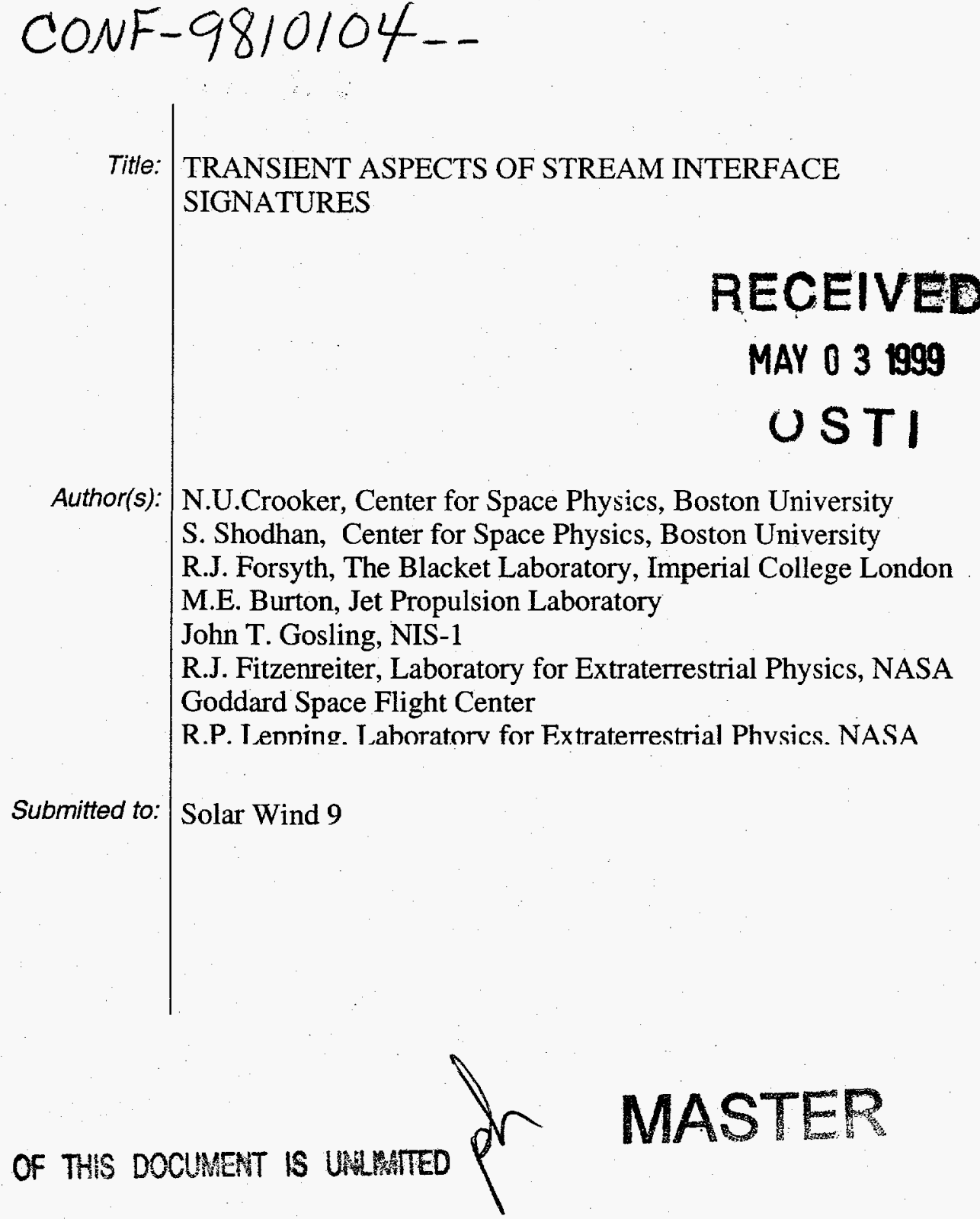

\section{Los Alamos NATIONAL LABORATORY}

Los Alamos National Laboratory, an affirmative action/equal opportunity employer, is operated by the University of California for the U.S. Department of Energy under contract W-7405-ENG-36. By acceptance of this article, the publisher recognizes that the U.S. Government retains a nonexclusive, royalty-free license to publish or reproduce the published form of this contribution, or to allow others to do so, for U.S. Government purposes. Los Alamos National Laboratory requests that the publisher identify this article as work performed under the auspices of the U.S. Department of Energy. Los Alamos National Laboratory strongly supports academic freedom and a researcher's right to publish; as an institution, however, the Laboratory does not endorse the viewpoint of a publication or guarantee its technical correctness. 


\section{DISCLAIMER}

This report was prepared as an account of work sponsored by an agency of the United States Government. Neither the United States Government nor any agency thereof, nor any of their employees, make any warranty, express or implied, or assumes any legal liability or responsibility for the accuracy, completeness, or usefulness of any information, apparatus, product, or process disclosed, or represents that its use would not infringe privately owned rights. Reference herein to any specific commercial product, process, or service by trade name, trademark, manufacturer, or otherwise does not necessarily constitute or imply its endorsement, recommendation, or favoring by the United States Government or any agency thereof. The views and opinions of authors expressed herein do not necessarily state or reflect those of the United States Government or any agency thereof. 


\section{DISCLAIMER}

\section{Portions of this document may be illegible in electronic image products. Images are produced from the best available original document.}




\title{
Transient Aspects of Stream Interface Signatures
}

\author{
N. U. Crooker', S. Shodhan', R. J. Forsyth', M. E. Burton ${ }^{3}$ J. T. Gosling ${ }^{4}$, \\ R. J. Fitzenreiter ${ }^{5}$, and R. P. Lepping \\ ${ }^{1}$ Center for Space Physics, Boston University, 725 Commonwealth Avenue, Boston, MA 02215 \\ ${ }^{2}$ The Blackett Laboratory, Imperial College, London SW7 2BZ, UK \\ ${ }^{3}$ Jet Propulsion Laboratory, MS 169-506, 4800 Oak Grove Drive, Pasadena, CA 91109. \\ 'Los Alamos National Laboratory, MS D466, Los Alamos, NM 87545 \\ 'Laboratory for Extraterrestrial Physics, NASA Goddard Space Flight Center, Greenbelt, MD 20771
}

\begin{abstract}
Although stream interfaces are steady-state, corotating boundaries between slow and fasi solar wind, their signatures are sometimes associated with transient features. Here we illustrate two modes of association: interfaces trailing interplanetary coronal mass ejections (ICMEs) at $1 \mathrm{AU}$ and interfaces within ICMEs in the range 4-5 AU. The former are readily understood as boundaries between transient slow wind and steady-state fast wind, where the ICMEs add variability to the interface signatures. The latter are puzzling and may be related to evolution of interfaces.
\end{abstract}

\section{INTRODUCTION}

On a global scale, stream interfaces have been welldocumented as steady-state, corotating boundaries between slow solar wind flow from the vicinity of the helmet streamer belt that encircles the Sun and fast flow from coronal holes $(1,2)$. Interfaces are central features of the corotating interaction regions (CIRs) on the leading edges of high-speed streams and were first identified by a rise in proton temperature and drop in density, resulting in a rise in entropy. Later findings showing composition changes at interfaces confirm the idea that they are markers of the solar boundary between slow and fast flow $(3,4 ;$ also, see 2$)$. The composition changes occur on both the leading and trailing edges of highspeed streams. Recently, comparable entropy drops on the trailing edges have also been documented (5).

In what may seem like a contradiction to this steadystate view, observations show that some stream interface signatures are associated with transient structures. Here we present observations of interfaces associated with interplanetary coronal mass ejections (ICMEs), some in the form of magnetic clouds (6) and others identified by counterstreaming electrons $(7)$. The observations fall into two groups: interfaces 1) trailing and 2) within CMEs. Those in group 1 are consistent with the steady-state pattern described above, while those in group 2 require additional explanation.

\section{INTERFACES TRAILING MAGNETIC CLOUDS}

Stream interfaces trailing magnetic clouds have been particularly noticeable at $1 \mathrm{AU}$ in Wind dam near solar minimum. Documented examples include multiple interfaces trailing the October 1995 cloud (8) and an interface preceded by extraordinarily high density plasma from an erupting filament trailing the January 1997 cloud (9).

Figure 1 shows another case from Wind data of a stream interface trailing a cloud, in May 1996. The cloud interval, shaded in the figure, is characterized by the large-scale rotation in magnetic field latitude angle $\theta_{B}$ (top panel), strong magnetic field magnitude $B$ (third panel), and low proton thermal speed (bottom panel). Counterstreaming electrons, signaling closed magnetic fields of ICMEs, roughly coincided with the first $80 \%$ of the cloud interval, through the end of May 29.

The stippled interval in Figure 1 at the end of the cloud highlights the location of the stream interface (SI). In this case, the SI is not a sharp boundary (cf. 2), and its characteristic signatures are not completely coincident. The density drop and thermal-speed rise (bottom panel) are irregular and extend a half day beyond the stippled interval to the end of the rising speed gradient. The flow deflection from west to east (fifth panel) begins within the cloud, at the start of the speed rise, and 


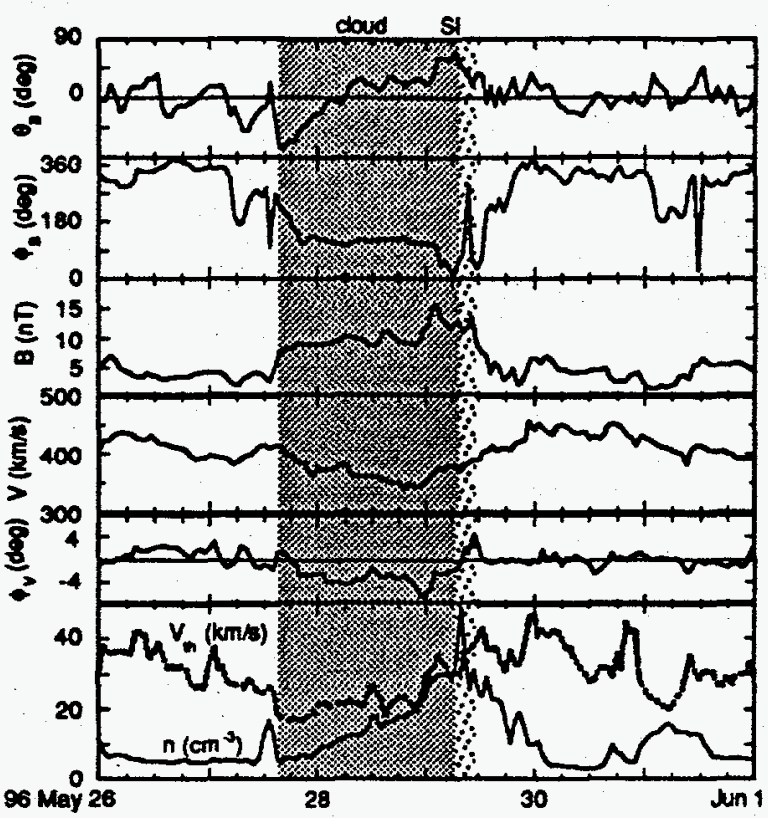

FIGURE 1. Time variations of magnetic latitude $\theta_{3}$, longitude $\phi_{3}$, and magnitude $B$, speed $V$, east-west flow angle $\$$, proton thermal speed $V_{\text {a. }}$ and density $n$ measured by the Wind spacecraft showing a stream interface (SI) on the trailing edge of a magnetic cloud.

ends with the stippled interval. Within the stippled interval is a sharp density peak marked by a sharp change in magnetic longitude $\phi_{B}$ (second panel) and a small dip in $B$. It is one of the high-density events analyzed by Shodhan et al. (10) and attributed to a transient pressure balance structure caught up in the CIR. It rides on the crest of a gradual density rise that builds from the low density at the cloud's leading edge. At the cloud's trailing edge, the characteristic steplike rise in thermal speed occurred twice, first at the end of May 29, coincident with the end of counterstreaming electrons (suggesting an alternative cloud boundary), and then at the end of the cloud, forming part of the SI signature. Variations of this mix of cloud and SI signatures are apparent in other cases of SIs trailing clouds $(8,9)$.

On a global scale, a stream interface trailing a cloud is consistent with the steady-state view of CIRs if one replaces steady slow flow with transient slow flow. Since most CMEs arise from the helmet streamer belt, they are rooted in the source of the slow solar wind. It is thus not surprising to encounter an ICME as slow flow corotates past a spacecraft. The deviation of the interface signatures from the ideal case can then be attributed to the presence of the ICME.

In the case of Figure 1, the global geometry appears to differ somewhat from encounter with an ICME in a band of slow flow corotating past a spacecraft, since one expects the slow flow to be sandwiched between highspeed streams of opposite polarity. Preliminary flux rope modeling of the cloud and comparison with the corresponding source surface map of coronal fields suggest that the slow flow in Figure 1 (only slightly slower than the not-so-fast flow following it) was associated in its entirety with an intrusion of the magnetic cloud from the north, inserting its away polarity into a toward sector. This view underscores the variability that can result from the interplay between transient and conotating structures.

\section{INTERFACES WITHIN ICMES}

Whereas stream interfaces trailing ICMEs fit naturally into the global CIR pattern, interfaces within ICMEs do not. Yet interface signatures have been observed within ICMEs identified by counterstreaming electrons near 4-5 AU. Specifically, in a survey of the first eight recurrent CIRs encountered by Ulysses in 1992-1993, four contained interfaces within ICMEs. These cases are known as CIRs 3,4,5, and 6, on the leading ediges of the correspondingly numbered high-speed streams (11).

Figure 2 shows data from CIR 6. The outer vertical lines mark the shocks bounding the CIR. The staded regions between them identify two closely-spaced counterstreaming electron events, the second of which contains the interface, marked by a thick line. The interface was identified by a drop in density and minor rise in temperature, resulting in a small rise in entropy (bottom panel), and by a coincident change in composition (3). The dashed line on the trailing boundary of the second counterstreaming event marks a major change in entropy owing in part to a sharp rise in temperature, typical of ICME boundaries, as discussed in the previous section. This entropy change had no accompanying composition signature and, hence, was not classified a an interface by Wimmer-Schweingruber et al. (3).

The magnetic field angles in Figure 2 show a change in magnetic polarity effected by a lange-scale field rocation, first analyzed by Lanzerotti et al. (12). The rotation spans all of the first and half of the second counterstreaming interval. Together with the elevated field magnitude and depressed temperature, these signatures indicate that the sector boundary was carried by a magnetic cloud. Similar cases have been documented by Crooker et al. (13). As in the case of the magnetic cloud in Figure 1, the field rotation ends at the stream interface, even though the interface is imbedded in the middle of a counterstreaming event which ends with a sharp temperature (and entropy) rise. These mixed signatures imply an ICME structure more complex than a single flux rope $(13,14,15)$. The point we wish to emphasize here, however, is that what would otherwise be taken for a corotating signature, that is, the stream interface signature, appears to be located inside a transient structure. 

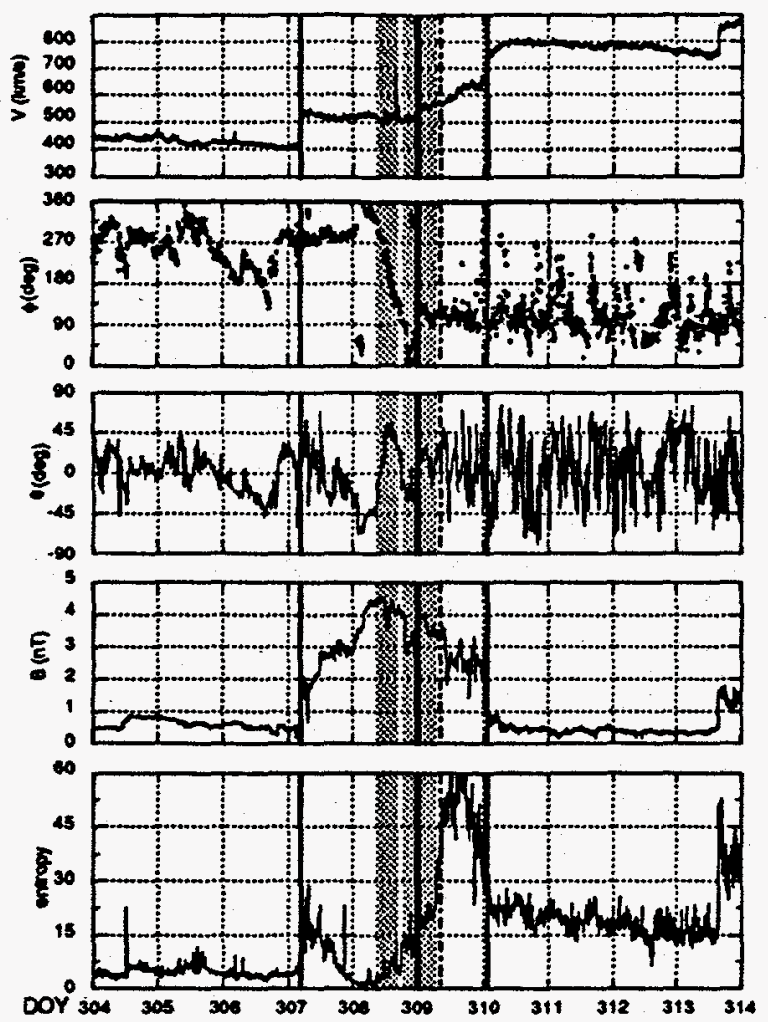

FIGURE 2. Time variations of speed $V$, magnetic longitude $\phi$, latitude $\theta$, and magnitude $B$, and entropy parameter $T / h^{1 / 2}$, where $T$ is temperature and $n$ is density, measured by Ulysses in 1992 (CIR 6). Thin vertical lines mark the forward and reverse CIR shocks, shading marks intervals of counterstreaming electrons, the second of which contains a stream interface marked by a thick line, and the dot-dash line marks a change in entropy.

Energetic particle data for the event in Figure 2 also reflect a mix of corotating and transient aspects. Lanzerotti et al (12), cognizant only of the ICME that cccurred days later (driving the unmarked shock on day 313 in Figure 2), pointed out a curious reverse in direction of 0.5-1.0 Mev protons at the sector boundary. Here we can associate that flow reversal with the ICME in the CIR. The flow reversal, coincident with the field rotation, appears to end at the interface. Preliminary plots of the flux of these energetic protons (R. G. Marsden, private communication, 1998) indicate that the interface also marks the sharp rise associated with particles energized at the reverse shock. This pattern was first identified as a corotating feature by Intriligator and Siscoe (16) in Pioneer data and confirmed by Intriligator et al. (17) in Ulysses data from other CIRs in the recurrent series. Thus the particle flux profile for the Figure 2 case is the same as if no ICME were present.

Crooker and Intriligator (18) reported on a similar energetic particle profile associated with an ICME in Pioneer 11 data, also near $5 \mathrm{AU}$. In that case, the interface occurred within the field rotation signaling the magnetic cloud rather than on its trailing edge.

The three additional cases of interfaces within ICMEs in Ulysses data, CIRs 3,4, and 5, have variations of the pattem shown in Figure 2. In each case, the sector boundary was carried by an ICME, marked by counterstreaming electrons and field rotations, but the position of the interface, as identified by WimmerSchweingruber et al. (3), relative to the field rotation and counterstreaming boundaries varies from case to case. CIR 3 is nearly like CIR 6, CIR 4 has multiple interfaces, two of which lie within a second counterstreaming interval well beyond the sector boundary, and CIR 5 has an interface near the end of a counterstreaming event but in the middle of a complex field rotation. Both CIR 3 and CIR 5 have secondary entropy changes following the last counterstreaming event, as in Figure 2. The energetic particle signatures relative to the ICMEs and interfaces have yet to be analyzed for these cases. Overall, the ICMEs lend a high level of variability to these cases, but all have in common interfaces imbedded in counterstreaming events.

Any attempt to explain interfaces within ICMEs must be tempered with the possibility that the interfaces have been misidentified or that the counterstreaming electrons do not signal an ICME. In the case of the interfaces, one could argue that they are not true interfaces because the flow deflection signatures are uncharacteristic. For example, in CIR 6, there is a deflection in the expected sense at the interface, but it is a small variation superposed upon a larger, more gradual deflection; in CIR 3 , there is essentially no deflection at the interface. In the case of the counterstreaming events, there is always the possibility that the beckstreaming electrons were generated locally (e.g., 19). Further, the counterstreaming signals at $5 \mathrm{AU}$ are often questionable, although for this study they were selected and confirmed by two authors.

On the other hand, since the interfaces within ICMEs identified here already differ from corotating interfaces in their association with transients, yet have many characteristics of corotating interfaces, it seems reasonable to propose that those in ICMEs are related to and possibly evolving into corotating interfaces.

\section{CONCLUSIONS}

We conclude with two interpretive sketches of stream interfaces related to ICMEs. Figure $3 a$ shows a corotating interface trailing an ICME. It serves as a boundary between fast flow and transient rather than steady-state slow flow. The sketch corresponds to cases of interface signatures trailing magnetic clouds, as in Figure 1, which have occurred repeatedly in Wind data at 1 AU near solar minimum.

Figure $3 b$ shows an interface within an ICME and an additional entropy change between the ICME and the 
a
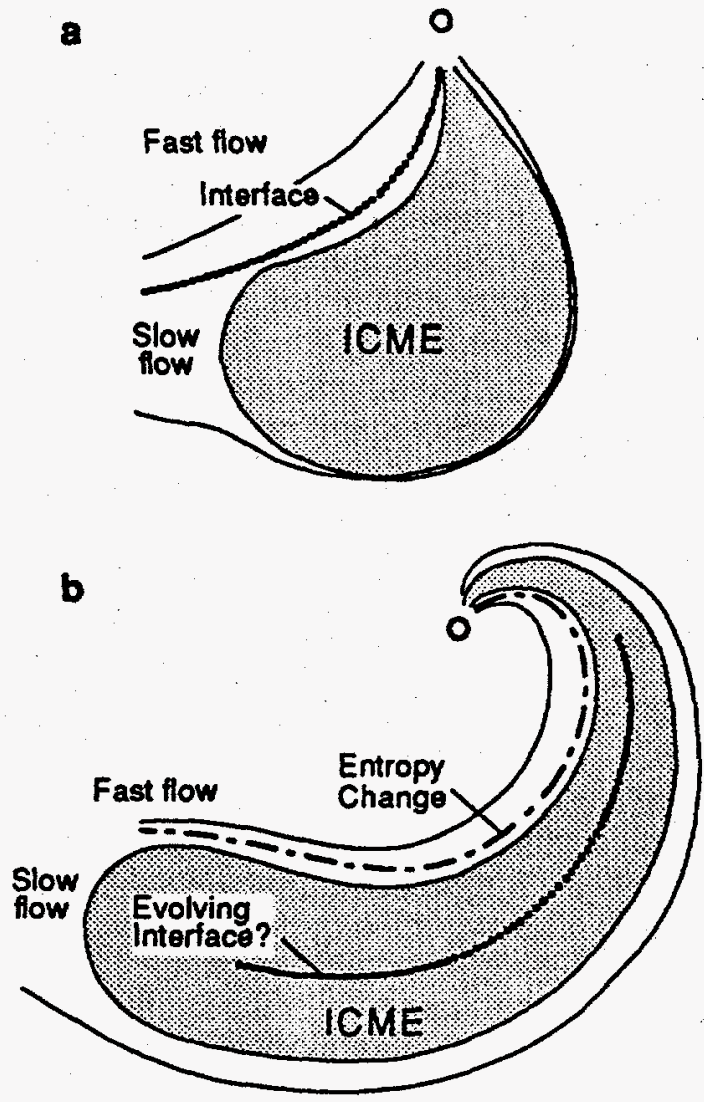

FIGURE 3. Sketches of a) interfaces trailing ICMEs near $1 \mathrm{AU}$ and b) interfaces within ICMEs beyond $1 \mathrm{AU}$.

fast flow. In contrast to Figure 3a, the ICME in Figure 3 b should have a more complex structure, a feature the figure lacks. Figure $3 \mathrm{~b}$ corresponds to cases of interfaces within counterstreaming electron events, as in Figure 2, which have been found in Ulysses data near 4-5 AU during the declining phase of the solar cycle. Whether the difference between interface position in Figure $2 \mathrm{a}$ and Figure $2 \mathrm{~b}$ is related to distance from the Sun or solar cycle phase is an open question.

In general, the results shown here demonstrate that stream interfaces, features normally attributed to steadystate, corotating structures, can at times have transient aspects. In particular, they can trail or be found within ICMEs. This association most likely contributes to the well-known variability of interface signatures (e.g., 2). One might argue that the interfaces associated with transients are 100 variable to be classified as such, but their similarity to the ideal case suggests a strong relationship to "true" interfaces and invites further analysis.

\section{ACKNOWLEDGMENTS}

This research was supported by NASA under grants NAG5-6658 and NAG5-7049 and by NASA/GSFC under PR S-96491-Z. Work at Los Alamos was performed under the auspices of the U.S. Department of Energy, also with support from NASA.

\section{REFERENCES}

1. Burlaga, L.F., J. Geophys. Res. 79, $3717-3725$ (1974).

2. Gosling, J.T., Asbridge, J.R., Bame, S.J., and Feldman, W.C., J. Geophys. Res. 83, 1401-1412 (1978).

3. Wimmer-Schweingruber, R.F., von Steiger, $R_{\text {., }}$ and Paerli, R., J. Geophys. Res. 102, 17,407-17,417 (1997).

4. Geiss. J., Gloectler, G., and von Steiger, R., Space Sci. Rev. 72, 49.60 (1995).

5. Burton, M.E., Neugebauer, M., N.U. Crooker, von Steiger, R., and E.J. Smith, J. Geophys. Res., in press (1998).

6. Burlaga, L.F., "Magnetic Clouds," in Physics of the Inner Heliosphere II, edited by R. Schwenn and E. Marsch, Springer-Verlag, New York, 1991, pp. 1-22.

7. Gosling, J.T, Baker, DN, Bame, S.J., Feldman, W.C., and Zwickl, R.D., J. Geophys. Res. 92, 8519. 8535 (1987).

8. Lepping, R.L., Burlage, L.F., Szabo, A., Ogilvie, K.W., Mish, W.H., Vassiliadis, D., Lazanus, A.J., Steinberg, J.T., Farrugia, C.J., and Janoo, L., $J$. Geophys. Res. 102, 14,049-14,063 (1997).

9. Burlaga, L.F., Fitzenreiter, R.J., Lepping, R.P., Ogilvie, K.W., Szabo, A., Lazarus, A.J. Steinberg. J.T., Howard, R.A. Michels, D., Farrugin, C., Lin, R.P., and Larson, D.E., J. Geophys. Res. 103, 277. 285 (1998).

10.Shodhan, S., Crooker, N.U., Fitzenreiter, R.J., Lepping, R.P., and Steinberg, J.T, "Density Enhancements in the Solar Wind," this volume.

11.Bame S.J., Goldstein, B.E. Gosling, J.T., Harvey, J.W., McComas, D.J., Neugebauer, M., and Phillips, J.L., Geophys. Res. Lett. 20, 2323-2326 (1993).

12.Lanzerotti, L.J., Maclennan, C.G., and Forsyth, R.J., Adv. Space Res. 17, (4/5)307-(4/5)310 (1996).

13.Crooker, N.U., Gosling, J.T., and Kahler, S.W., J. Geophys, Res. 103, $301-306$ (1998).

14.Kahler, S.W., Crooker, N.U., and Cosling, J.T., "Exploring ISEE-3 Magnetic Cloud Polarities with Electron Heat Fluxes," this volume.

15.Vandes, M., and Karlicky, M., "Double Flux Rope Structure of Magnetic Clouds," this volume.

16.Intriligator, D.S., and Siscoe, G.L. Geophys. Res. Lett. 21, 1117-1120 (1994).

17.Intriligator, D.S., Siscoe, G.L., Wibberenz, G., Kunow, H., and Gosling, J.T., Geophys. Res. Lett. 22, 11731176 (1995).

18.Crooker, N.U., and Intriligator, D.S., "A Magnetic Cloud at Pioneers 10 and 11: Relation to Heliospheric Current Sheet, Stream Interface, and Energetic Ions," in Solar Wind Eight, edited by D. Winterhalter et al., New York, AIP Conf. Proc. 4382,1996, pp. 442-444.

19.Gosling, J.T., Bame, S.J., Feldman, W.C., McComas, D.J. Phillips, J.L. and Goldstein, B.E., Geophys. Res. Lett. 20, 2335-2338 (1993). 